\title{
Corrections to the Entropy in Higher Order Gravity
}

\author{
Luis Alejandro Correa-Borbonet \\ Departamento de Física \\ Universidade Federal de Mato Grosso, \\ Av. Fernando Corrêa da Costa, s/no-Bairro Coxipó \\ 78060-900-Cuiabá-MT, Brazil \\ (Received on 14 October, 2005)
}

\begin{abstract}
Thermal corrections to the entropy of black holes in the Lovelock gravity are calculated. As the thermodynamic behavior of the black holes of this theory falls into two classes, the thermodynamic quantities are computed in each case. Finally, the logarithmic prefactors are obtained in two different limits.
\end{abstract}

\section{INTRODUCTION}

During the late 1960's and early 1970's an intense research activity in the field of black hole physics lead to the discovery of the laws of black hole mechanics. Soon, it was realized a striking resemblance with the laws of thermodynamics[1, 2]. In this context Bekenstein proposed that the entropy in black holes is proportional to the surface area of the event horizon. Moreover, Hawking found that black holes generate also a thermal radiation, due to quantum pair production in their gravitational potential gradient and the presence of the event horizon. The temperature of this radiation is given in terms of the surface gravity at the event horizon, $T_{H}=\hbar \kappa / 2 \pi$. An immediate consequence of this identification of the temperature is that the proportionality constant between the entropy and the area is fixed, $S_{B H}=A / 4 G$.

Althought this knowledge about the black hole thermodynamics is well established, we do not have a complete answer to this question in the statistical mechanics framework. However, there has been a substantial progress in identifying the microscopic degrees of freedom responsible for the Bekenstein-Hawking entropy. This advance has come from string theory[4] and loop quantum gravity[5]. In the case of string theory it is present a massless spectrum including the graviton, and at low energy it gives supergravity effectives theories. Black Holes therefore appear as classical solutions of low energy string theory.

The next step in this line of research has been the study of the leading correction to entropy. A common characteristic of the different approaches is the proportionality to $\ln S_{B H}$ [6],[7],[8]. However, the proportionality constant does not exhibit the same universality. It can be shown that logarithmic corrections to thermodynamic entropy arise in all thermodynamic systems when small stable fluctuations around equilibrium are taken into account[9]. The stability condition is equivalent to the specific heat being positive.

On the other hand the study of the thermodynamic properties of black holes has been extended to higher order gravity theories[10]. Within these theories there is a special class of gravitational actions, of higher order in the curvature, known as Lovelock gravity [11]. Lovelock gravity is exceptional in the sense that although it contains higher powers of the curvature in the Lagrange density, the resulting equations of motion contain no more than second derivatives of the metric. It is also a covariant and ghost free theory as it happens in the case of Einstein's General Relativity.

An important result that was found in the thermodynamic context is that the area law is a peculiarity of the EinsteinHilbert theory [12]. These facts motivates a deeper study of the thermodynamics of the black hole solutions of such exotic theories [13][14],[15]. In this paper we will study the corrections to the entropy for the black holes solutions of Lovelock gravity. We shall first briefly review such a formulation.

\section{HIGHER DIMENSIONAL GRAVITY}

The Lanczos-Lovelock action is a polynomial of degree $[d / 2]$ in the curvature, which can be expressed in the language of forms as [12]

$$
I_{G}=\kappa \int \sum_{m=0}^{[d / 2]} \alpha_{m} L^{(m)},
$$

where $\alpha_{m}$ are arbitrary constants, and $L^{(m)}$ is given by

$$
L^{(m)}=\varepsilon_{a_{1} \cdots a_{d}} R^{a_{1} a_{2}} \cdots R^{a_{2 m-1} a_{2 m}} e^{a_{2 m+1}} \cdots e^{a_{d}},
$$

with $R^{a b}$ being the Riemann curvature two-forms given by

$$
R^{a b}=d \omega^{a b}+\omega_{c}^{a} w^{c b} .
$$

Here $w_{a b}$ are the spin connection one-forms and $e^{a}$ the vielbein. A wedge product between forms is understood throughout.

The corresponding field equations can be obtained varying with respect to $e^{a}$ and $w^{a b}$. In [12] the expression for the coefficients $\alpha_{m}$ was found requiring the existence of a unique cosmological constant. In such a case these theories are described by the action

$$
I_{k}=\kappa \int \sum_{p=0}^{k} c_{p}^{k} L^{(p)},
$$

which corresponds to (1) with the choice

$$
\alpha_{p}:=c_{p}^{k}= \begin{cases}\frac{l^{2(p-k)}}{(d-2 p)}\left(\begin{array}{l}
k \\
p
\end{array}\right), & , p \leq k \\
0 & , p>k\end{cases}
$$

for the parameters, where $1 \leq k \leq[(d-1) / 2]$. For a given dimension $d$, the coefficients $c_{m}^{\bar{k}}$ give rise to a family of inequivalent theories, labeled by $k$ which represent the highest power 
of curvature in the Lagrangian. This set of theories possesses only two fundamental constants, $\kappa$ and $l$, related respectively to the gravitational constant $G_{k}$ and the cosmological constant $\Lambda$ through

$$
\begin{aligned}
& \kappa=\frac{1}{2(d-2) \Omega_{d-2} G_{k}}, \\
& \Lambda=-\frac{(d-1)(d-2)}{2 l^{2}} .
\end{aligned}
$$

For black hole solutions that are asymptotically flat we consider the vanishing cosmological constant limit case. When $l \rightarrow \infty$ the only non-vanishing terms in $\mathrm{Eq}(4)$ is the kth one; therefore the action is obtained from $\mathrm{Eq}(1)$ with the choice of coefficients

$$
\alpha_{p}:=\tilde{c}_{p}^{k}=\frac{1}{(d-2 k)} \delta_{p}^{k}
$$

in this case the action reads

$$
\tilde{I}_{k}=\frac{\kappa}{(d-2 k)} \int \varepsilon_{a_{1} \cdots a_{d}} R^{a_{1} a_{2}} \cdots R^{a_{2 k-1} a_{2 k}} e^{a_{2 k+1} \cdots e^{a_{d}}} .
$$

Note that for $k=1$ the Einstein action without cosmological constant is recovered, while for $k=2$ we obtained the GaussBonnet action,

$I_{2}=\frac{(d-2) ! \kappa}{(d-4)} \int d^{d} x \sqrt{-g}\left(-R_{\mu v \alpha \beta} R^{\mu v \alpha \beta}+4 R_{\mu v} R^{\mu v}-R^{2}\right)$.

Returning to the action (4) we remember that this set of theories possess asymptotically AdS black hole solutions given by[12],

$$
\begin{aligned}
d s^{2}= & -\left(1+\frac{r^{2}}{l^{2}}-\left(\frac{2 G_{k} M+\delta_{d-2 k, 1}}{r^{d-2 k-1}}\right)^{1 / k}\right) d t^{2}+ \\
& \frac{d r^{2}}{1+\frac{r^{2}}{l^{2}}-\left(\frac{2 G_{k} M+\delta_{d-2 k, 1}}{r^{d-2 k-1}}\right)^{1 / k}}+r^{2} d \Omega_{d-2}^{2} .
\end{aligned}
$$

The black hole mass for any value of $k$ is a monotonically increasing function of the horizon radius $r_{+}$, which reads

$$
M\left(r_{+}\right)=\frac{r_{+}^{d-2 k-1}}{2 G_{k}}\left(1+\frac{r_{+}^{2}}{l^{2}}\right)^{k}-\frac{1}{2 G_{k}} \delta_{d-2 k, 1} .
$$

The presence of the Kronecker delta within the metrics (11) signals the existence of two possible black hole vacua $(M=0)$ with different causal structures. The generic case, with $d-$ $2 k \neq 1$, is

$$
\begin{aligned}
d s^{2}= & -\left(1+\frac{r^{2}}{l^{2}}-\left(\frac{2 G_{k} M}{r^{d-2 k-1}}\right)^{1 / k}\right) d t^{2}+ \\
& \frac{d r^{2}}{1+\frac{r^{2}}{l^{2}}-\left(\frac{2 G_{k} M}{r^{d-2 k-1}}\right)^{1 / k}}+r^{2} d \Omega_{d-2}^{2}
\end{aligned} .
$$

Analogously with the Schwarzschild-AdS metric, this set possesses a continuous mass spectrum, whose vacuum state is the AdS spacetime. The other case is obtained for odd dimensions, and it is a peculiarity of Chern-Simons theories. From (11) we obtain,

$$
\begin{aligned}
d s^{2}= & -\left(1+\frac{r^{2}}{l^{2}}-\left(2 G_{k} M+1\right)^{1 / k}\right) d t^{2}+ \\
& \frac{d r^{2}}{1+\frac{r^{2}}{l^{2}}-\left(2 G_{k} M+1\right)^{1 / k}}+r^{2} d \Omega_{d-2}^{2} .
\end{aligned}
$$

Here, the black hole vacuum differs from AdS spacetime.

\section{CANONICAL FORMALISM}

In this section we review [9] the derivation of the entropy in the canonical formalism. Looking for the entropy corrections it is considered the existence of small thermal fluctuations around the equilibrium. Then, we begin with the canonical partition function

$$
Z(\beta)=\int_{0}^{\infty} \Omega(E) e^{-\beta E} d E
$$

where $\Omega(E)$ is the density of states, that can be obtained from the partition function doing an inverse Laplace transform

$$
\Omega(E)=\frac{1}{2 \pi i} \int_{c-i \infty}^{c+i \infty} Z(\beta) e^{\beta E} d \beta=\frac{1}{2 \pi i} \int_{c-i \infty}^{c+i \infty} e^{S(\beta)} d \beta,
$$

where

$$
S(\beta)=\ln Z(\beta)+\beta E
$$

is the entropy. The integral can be performed by the method of steepest descent around the saddle point $\beta_{0}=1 / T_{0}$ such that $S_{0}^{\prime}=(\partial S / \partial \beta)_{\beta=\beta_{0}}=0$. Here $T_{0}$ is the equilibrium temperature. Expanding the entropy around $\beta_{0}$, we have

$$
S=S_{0}+\frac{1}{2}\left(\beta-\beta_{0}\right)^{2} S_{0}^{\prime \prime}+\ldots
$$

Substituting (18) in (16) and integrating we obtain

$$
\Omega(E)=\frac{e^{S_{0}}}{\sqrt{2 \pi S_{0}^{\prime \prime}}} .
$$

Finally, using the Boltzmann's formula, is obtained

$$
S=\ln \Omega=S_{0}-\frac{1}{2} \ln \left(S_{0}^{\prime \prime}\right)+\ldots
$$

Here $\mathcal{S}$ is the entropy at equilibrium. This is to be distinguished from the function $S(\beta)$, which is the entropy at any temperature.

The logarithmic term can be transformed taking into account that $S_{0}^{\prime \prime}$ is the fluctuation of the mean squared energy, i.e,

$$
S_{0}^{\prime \prime}=<E^{2}>-<E>^{2}
$$


and that the specific heat is $C=(\partial E / \partial T)_{T_{0}}$. Therefore

$$
\mathcal{S}=S_{0}-\frac{1}{2} \ln \left(C T^{2}\right) \text {. }
$$

This result apply to stable thermodynamic systems with small fluctuations around the equilibrium. The stability condition is equivalent to the specific heat being positive. On the other hand is assumed that the quantum fluctuations of the thermodynamics quantities under consideration are small. In other words, for black holes very close to extremality $(T \rightarrow 0)$, the fluctuation analysis ceases to be valid due to large quantum fluctuactions[9].

\section{CORRECTIONS TO THE ENTROPY IN HIGHER ORDER GRAVITY}

\section{A. Asymptotically AdS black hole solutions}

Reviewing the thermodynamic properties of the black hole solution (13) we began with the expression for the Hawking temperature, that is

$$
T_{H}=\frac{1}{4 \pi k_{B} k}\left((d-1) \frac{r_{+}}{l^{2}}+\frac{d-2 k-1}{r_{+}}\right),
$$

where $r_{+}$is the horizon radius. Note that for all $k$ such that $d-2 k-1 \neq 0$ the temperature has the same behavior that the Schwarzschild-AdS black hole $(\mathrm{k}=1)$, that is: the temperature diverges at $r_{+}=0$. Also has a minimum at $r_{c}$ given by

$$
r_{c}=l \sqrt{\frac{d-2 k-1}{d-1}}
$$

and grows linearly for large $r_{+}$. Consequently we can calculate the specific heat $C_{k}=\frac{\partial M}{\partial T}$ as a function of $r_{+}$. Using (23) and (12) we obtain,

$$
C_{k}=k \frac{2 \pi k_{B}}{G_{k}} r_{+}^{d-2 k}\left(\frac{r_{+}^{2}+r_{c}^{2}}{r_{+}^{2}-r_{c}^{2}}\right)\left(1+\frac{r_{+}^{2}}{l^{2}}\right)^{k-1} .
$$

Here the function $C_{k}$ has an unbounded discontinuity at $r_{+}=$ $r_{c}$, signaling a phase transition. We will deal with black hole with horizon radius that satisfies the condition $r_{+}>r_{c}$, where the specific heat is positive and the correction formula (22) can be apply.

Finally we present the entropy function

$$
S_{k}=k \frac{2 \pi k_{B}}{G_{k}} \int_{0}^{r_{+}} r^{d-2 k-1}\left(1+\frac{r^{2}}{l^{2}}\right)^{k-1} d r,
$$

obtained from the Euclidean path integral formalism[12]. Similar results are obtained in the Lagrangian formalism [16].

For simplicity we just perform the calculations for black holes with $k=2$. Therefore, for the entropy we get

$$
S_{2}^{(0)}=\frac{4 \pi k_{B}}{G_{2}} r_{+}^{d-4}\left[\frac{1}{(d-4)}+\frac{r_{+}^{2}}{(d-2) l^{2}}\right]
$$

where (0) stands for the uncorrected entropy. In terms of this entropy the Hawking temperature and the specific heat are given by:

$$
\begin{aligned}
& T_{H}= {\left[\frac{1}{8 \pi k_{B} l^{2}}\left(\frac{4 \pi k_{B}}{G_{2} l^{2}}\right)^{-1 /(d-2)}\right] \times } \\
& \times \frac{\left[(d-1)+\frac{(d-5) l^{2}}{r_{+}^{2}}\right]}{\left[\frac{l^{2}}{(d-4) r_{+}^{2}}+\frac{1}{d-2}\right]^{1 /(d-2)}}\left(S_{2}^{(0)}\right)^{1 /(d-2)}, \\
& C_{2}=\left(\frac{r_{+}^{2}+r_{c}^{2}}{r_{+}^{2}-r_{c}^{2}}\right)\left(1+\frac{l^{2}}{r_{+}^{2}}\right) \frac{1}{\frac{l^{2}}{(d-4) r_{+}^{2}}+\frac{1}{(d-2)}} S_{2}^{(0)} .
\end{aligned}
$$

In the limit $\mathrm{r}_{+} \gg l, C_{2}$ approaches the value

$$
C_{2}=(d-2) S_{2}^{(0)}
$$

and for the entropy we get

$$
S_{2}=S_{2}^{(0)}-\frac{d}{2(d-2)} \ln S_{2}^{(0)}+\ldots
$$

This result is identical to the one obtained for the AdSSchwarzschild black holes[9].

We can also study the correction to the entropy near the transition point $r_{c}$ (after the minimum of $C_{2}$ ). Assuming $r_{+}^{2}-$ $r_{c}^{2} \approx l^{2}$ (small $\left.l\right)$, the entropy behaves as

$$
S_{2}^{(0)}=\frac{4 \pi k_{B}}{G_{2}} r_{+}^{d-4}\left[\frac{1}{(d-4)}+\frac{2(d-3)}{(d-2)(d-1)}\right] .
$$

Consequently the relations between the thermodynamic quantities are

$$
\begin{gathered}
T_{H}=\frac{B}{A^{1 /(d-4)}}\left(S_{2}^{(0)}\right)^{1 /(d-4)}, \\
C_{2}=\frac{C}{A} S_{2}^{(0)},
\end{gathered}
$$

where

$$
\begin{gathered}
A=\frac{4 \pi k_{B}}{G_{2}}\left[\frac{1}{(d-4)}+\frac{2(d-3)}{(d-2)(d-1)}\right], \\
B=\frac{1}{8 \pi k_{B}} \frac{1}{l^{2}}\left[(d-1)+\frac{(d-5)(d-1)}{2(d-3)}\right], \\
C=\frac{4 \pi k_{B}}{G_{k}}\left[1+\frac{2(d-3)}{d-1}\right]\left[\frac{2(d-3)}{(d-1)}+\frac{(d-5)}{(d-1)}\right] .
\end{gathered}
$$

Therefore

$$
\mathcal{S}_{2}=S_{2}^{(0)}-\frac{d-2}{2(d-4)} \ln S_{2}^{(0)}+\ldots
$$

It is interesting to note that the entropy correction in this limit is greater than the entropy correction found in (31). 


\section{B. Chern-Simons Black Holes}

Now let us consider the Chern-Simons black holes with metric (14). The thermodynamic quantities can be obtained considering $d-2 k-1=0$. So,

$$
\begin{gathered}
T_{H}=\frac{1}{4 \pi k_{B} k}\left((d-1) \frac{r_{+}}{l^{2}}\right), \\
C_{C S}=k \frac{2 \pi k_{B}}{G_{k}} r_{+}\left(1+\frac{r_{+}^{2}}{l^{2}}\right)^{k-1},
\end{gathered}
$$

and

$$
S_{k}=k \frac{2 \pi k_{B}}{G_{k}} \int_{0}^{r_{+}}\left(1+\frac{r^{2}}{l^{2}}\right)^{k-1} d r .
$$

In this case the temperature is not divergent and the specific heat is a continuous monotonically increasing positive function of $r_{+}$

In the limit $r_{+} \gg l$ we get

$$
C_{C S}=(2 k-1) S_{k}^{0}
$$

and

$$
S_{k}=S_{k}^{(0)}-\frac{2 k+1}{2(2 k-1)} \ln S_{k}^{(0)}+\ldots
$$

Note that in this limit the results, as was expected, are similar to those found in the previous section.
On the other hand, for $r_{+} \approx l$, we obtain

$$
S_{k}=S_{k}^{(0)}-\frac{3}{2} \ln S_{k}^{(0)}+\ldots
$$

Similar to the proportionality constant found in [9] for the BTZ black hole[17].

\section{CONCLUSIONS.}

In this paper we have calculated the entropy corrections for different kinds of black holes in the context of higher order gravity. The results are similar to those found in the literature despite the fact that the area law is not satisfied. Also is confirmed the lack of universality of the logarithmic prefactor. In the case of Chern-Simons black holes with small horizon radius we have found that the logarithmic prefactor does not depended of the dimension.

\section{Acknowledgment}

The author thanks Prof. Sandro Silva e Costa at the Physics Department-UFMT for hospitality. This work was supported by Fundação de Amparo à Pesquisa do Estado de Mato Grosso (FAPEMAT) and Conselho Nacional de Desenvolvimento Científico e Tecnológico $(\mathrm{CNPq})$. The authors also thank the organizers of the Conference "100 years of Relativity" where these results were presented.
[1] J. D. Bekenstein, Phys. Rev. D 7, 2333 (1973); Phys. Rev. D 9 , 3292 (1974).

[2] S. Hawking, Phys. Rev. Lett. 26, 1344 (1971) ; Nature 248, 30 (1974); Commun. Math. Phys. 43, 199 (1975).

[3] S. W. Hawking and D. Page, Comm. Math. Phys. 87, 577 (1983).

[4] A. Strominger and C. Vafa, Phys. Lett. B379, 99 (1996).

[5] A. Ashtekar, J. Baez, A. Corichi, and K. Krasnov, Phys. Rev. Lett. 80, 904 (1998).

[6] R. K. Kaul and P. Majumdar, Phys. Rev. Lett. D84, 5255 (2000).

[7] S. Carlip, Class.Quant.Grav. 17, 4175 (2000).

[8] S. N. Solodukhin, Phys. Rev. D 57, 2410 (1998).

[9] S. Das, P. Majumdar, and R. Bhaduri, Class. Quant. Grav. 19, 2355 (2002).

[10] R. C. Myers and J. Z. Simon, Phys. Rev. D38, 2434 (1988) ; T. Jacobson, G. Kang, and R. C. Myers, Phys. Rev. D52, 3518 (1995); T. Jacobson and R. C. Myers, Phys. Rev. Lett. 70, 3684 (1993); R. C. Myers, gr-qc/9811042.

[11] D. Lovelock, J. Math. Phys. 12, 498 (1971).
[12] J. Crisostomo, R. Troncoso, and J. Zanelli, Phys. Rev. D 62, 084013 (2000).

[13] M. R. Setare, Phys. Lett. 573, 173 (2003); M. R. Setare, Eur. Phys. J. C33, 555 (2004); Mu-In Park, JHEP 0412, 041 (2004); Rong-Gen Cai, Phys. Lett. B 582, 237 (2004); Rong-Gen Cai, Phys. Rev. D 65, 084014 (2002); Rong-Gen Cai and KwangSup Soh, Phys. Rev. D 59, 044013 (1999); Rong-Gen Cai and Qi Guo, Phys. Rev. D 69, 104025 (2004); Shin'ichi Nojiri, Sergei D. Odintsov, and Sachiko Ogushi Phys. Lett. B 544, 237 (2002).

[14] Shin'ichi Nojiri, Sergei D. Odintsov, and Sachiko Ogushi, Phys. Rev. D65, 023521 (2002).

[15] E. Abdalla and L. Alejandro Correa-Borbonet, Phys. Rev. D 65, 124011 (2002).

[16] P. Mora, R. Olea, R. Troncoso, and J. Zanelli, JHEP 0406, 036 (2004).

[17] M. Banados, C. Teitelboim, and J. Zanelli, Phys. Rev. Lett 69, 1849 (1992). 